\title{
THREE PARTIAL ORDERS ARISING FROM MULTIPLICATION ALTERATION BY TWO-COCYCLES
}

\author{
BY DAVE RIFFELMACHER
}

Communicated by James Bramble, December 8, 1975

1. Introduction. We introduce three partial orders arising from multiplication alteration by two-cocycles and show how some order properties of an algebra are related to its structure. Throughout this note $C$ is an (associative) algebra with unit 1 over a commutative ring $k, \sigma=\Sigma a_{i} \otimes b_{i} \otimes c_{i}$ in $C \otimes C \otimes C$ is a $C$-two-cocycle with unity element $e_{\sigma}$, and $C^{\sigma}$ is the $k$-algebra obtained from $C$ and $\sigma$ with product $x^{\sigma} * y^{\sigma}=\left(\Sigma a_{i} x b_{i} y c_{i}\right)^{\sigma}$. The reader is referred to [2] for the basic theory of multiplication alteration by two-cocycles. The author extends thanks to Moss Sweedler for directing this research.

2. Definitions. In this section we define three partial orders on the class of $k$-algebras which are due to Sweedler.

Definition 2.1. $C, D k$-algebras. $C$ Amitsur dominates $D$ if there is a $C$ two-cocycle $\sigma$ with $D \cong C^{\sigma}$ as $k$-algebras. $C$ is an Amitsur atom if $C$ Amitsur dominates $D$ implies $D \cong C$.

EXAMPLE. The $k$-algebra of two by two upper triangular matrices with entries in $k$ is an Amitsur atom.

Given a $C$-two-cocycle $\sigma$ and a $C^{\sigma}$-two-cocycle $\tau$, writing out $\left(x^{\sigma}\right)^{\tau} *\left(y^{\sigma}\right)^{\tau}$ suggests a candidate for a $C$-two-cocycle $\gamma$ with $\left(C^{\sigma}\right)^{\tau} \cong C^{\gamma}$ via $\left(x^{\sigma}\right)^{\tau} \leftrightarrow x^{\gamma}$. Direct calculation shows this element is a $C$-two-cocycle.

Proposition 2.2. Amitsur dominance is a partial order on the class of $k$ algebras.

We define a map $\varphi_{\sigma}: C^{\sigma} \otimes C^{\sigma o} \rightarrow C \otimes C^{\circ}$ by $\varphi_{\sigma}\left(x^{\sigma} \otimes y^{\sigma o}\right)=\Sigma a_{i} a_{j} x b_{j}$ $\otimes\left(c_{j} b_{i} y c_{i}\right)^{\mathbf{o}}$, where $C^{\mathbf{o}}$ is the opposite algebra of $C$. A straightforward calculation using the $C$-two-cocycle relations for $\sigma$ shows that $\varphi_{\sigma}$ is a $k$-algebra map. Let the change of rings functor induced by $\varphi_{\sigma}$ from the category $M(C)$ of $C$-bimodules to the category $M\left(C^{\sigma}\right)$ of $C^{\sigma}$-bimodules be denoted ( $)^{\sigma}$.

Definition 2.3. $C, D k$-algebras. $C$ Hochschild dominates $D$ if there is a $C$-two-cocycle $\sigma$ with $D \cong C^{\sigma}$ as $k$-algebras and ( $)^{\sigma}$ is dense. $C$ is a Hochschild atom if ( $)^{\sigma}$ is dense for all $C$-two-cocycles $\sigma$.

Proposition 2.4. Hochschild dominance is a partial order.

AMS (MOS) subject classifications (1970). Primary 16A48, 18H15; Secondary 16A16. 
Let $A(C)$ be the category of $k$-algebras over $C$. Define a functor $F^{\sigma}: A(C)$ $\rightarrow A\left(C^{\sigma}\right)$ by $F^{\sigma}(C \stackrel{f}{\longrightarrow} D)=C^{\sigma} \stackrel{f^{\sigma}}{\longrightarrow} D^{f(\sigma)}$, where $f^{\sigma}\left(a^{\sigma}\right)=f(a)^{f(\sigma)}$ and $f(\sigma)=$ $f^{\otimes_{3}}(\sigma)$, and

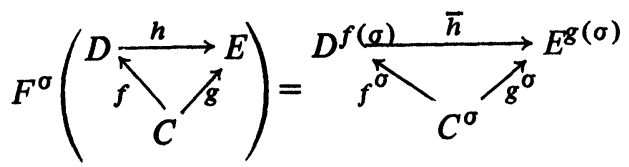

with $\bar{h}\left(d^{f(\sigma)}\right)=h(d)^{g(\sigma)}$.

Definition 2.5. $C, D k$-algebras. $C$ categorically dominates $D$ if there is a $C$-two-cocycle $\sigma$ with $D \cong C^{\sigma}$ as $k$-algebras and $F^{\sigma}: A(C) \rightarrow A\left(C^{\sigma}\right)$ is dense. $C$ is a categorical atom if $F^{\sigma}$ is dense for all $C$-two-cocycles $\sigma$.

Proposition 2.6. Categorical dominance is a partial order.

3. Three characterization theorems. We present one theorem to indicate how each of the partial orders from $\$ 2$ may be used to characterize a type of $k$ algebra. The first theorem provides the converse of a result of [2].

THEOREM 3.1. $k$ field. $C k$-algebra of $k$-dimension $n$. The following are equivalent:

(a) $C$ is a central simple k-algebra.

(b) $C$ Amitsur dominates all $k$-algebras of $k$-dimension $n$.

(c) $C$ Amitsur dominates $k \oplus \cdot \stackrel{n}{\cdot} \oplus *$ and $k[x] /\left(x^{n}\right)$.

(d) C Amitsur dominates a $k$-separable algebra and a $k$-purely inseparable (cf. [3]) algebra.

IndiCATION OF PROOF. (a) implies (b) is [2, Theorem 6.1]. The implication (d) $\Rightarrow$ (a) follows from the behavior of the center $Z(C)$ of $C$ and the Jacobson radical $J(C)$ of $C$ under multiplication alteration by two-cocycles.

Before stating the next two theorems, we recall a class of $C$-two-cocycles mentioned in [2].

ExAmple (WATERhouse). Let $B$ be a $k$-separable subalgebra of $C$ with separability idempotent $e$. Then $\sigma_{B}=e \otimes 1+1 \otimes e-(1 \otimes e)(e \otimes 1)$ is a $C$ two-cocycle with $e_{\sigma_{B}}=1$.

THEOREM 3.2. $k$ field. $C$ an algebraic $k$-algebra with nilpotent Jacobson radical $J(C)$ and $C / J(C)$ locully finite. The following are equivalent:

(a) ( $)^{\sigma}: M(C) \rightarrow M\left(C^{\sigma}\right)$ is an equivalence for all $\sigma$.

(b) $C$ is a Hochschild atom.

(c) All k-separable subalgebras of $C$ are central.

(d) $\varphi_{\sigma}$ is an isomorphism for all C-two-cocycles $\sigma$.

INDICATION OF PROOF. The implication (b) $\Rightarrow(\mathrm{c})$ follows from a study of the functors ( ) ${ }^{\sigma} B$ for Waterhouse two-cocycles. To show (c) implies (d), one 
proves that under the condition of (c) the hypothesis of the following lemma holds for all $\sigma$.

LEMMA 3.3. Let $\sigma=\Sigma a_{i} \otimes b_{i} \otimes c_{i}$ be a $C$-two-cocycle and

$$
z_{\sigma}=\sum a_{i} a_{j} \otimes_{Z(C)} b_{j}^{\circ} \otimes c_{j} b_{i} \otimes_{Z(C)} c_{i}^{\circ}
$$

in $C \otimes_{Z(C)} C^{\circ} \otimes C \otimes_{Z(C)} C^{\circ}$. If $z_{\sigma}$ is invertible, $\varphi_{\sigma}$ is an isomorphism.

THEOREM 3.4. $k$ field. $C$ an algebraic $k$-algebra with nilpotent Jacobson radical $J(C)$ and $C / J(C)$ locally finite. The following are equivalent:

(a) $F^{\sigma}: A(C) \rightarrow A\left(C^{\sigma}\right)$ is an equivalence for all $\sigma$.

(b) $C$ is a categorical atom.

(c) $C$ has no $k$-separable subalgebras (except $k$ ).

(d) All C-two-cocycles are invertible.

INDICATION OF PROOF. The implication $(\mathrm{b}) \Rightarrow(\mathrm{c})$ follows from a study of the functors $F^{\sigma}$ for Waterhouse two-cocycles. One proves (c) and (d) are equivalent using Wedderburn-Artin structure theory (cf. [1]) and the theory of purely inseparable algebras [3]. Then, after a reduction to the case $e_{\sigma}=1$, one shows that under the hypotheses of (c) and (d), the condition of the following lemma holds for all $C$-two-cocycles $\sigma$ with $e_{\sigma}=1$.

Lemma 3.5. Let $\sigma=\Sigma a_{i} \otimes b_{i} \otimes c_{i}$ be a C-two-cocycle with $e_{\sigma}=1$ and let

$$
\omega_{\sigma}=\sum\left(a_{i_{1}} a_{i_{2}} a_{i_{3}} a_{i_{4}}\right)^{\circ} \otimes b_{i_{4}} \otimes\left(c_{i_{4}} b_{i_{3}}\right)^{\circ} \otimes c_{i_{3}} b_{i_{2}} \otimes\left(c_{i_{2}} b_{i_{1}}\right)^{\circ} \otimes c_{i_{1}}
$$

in $\left(C^{\circ} \otimes C\right)^{\otimes}$. If $\omega_{\sigma}$ is invertible, there is a $C^{\sigma}$-two-cocycle $\tau$ with $F^{\tau} \circ F^{\sigma}=$ $F^{1 \otimes 1 \otimes 1}$.

4. Remark. If $\delta$ is any element of $C \otimes C \otimes C$, we may still define a functor $F^{\delta}$ on $A(C)$. Then the image of $F^{\delta}$ is in $A\left(C^{\delta}\right)$ iff $\delta$ is a $C$-two-cocycle. This provides an easy proof of Proposition 2.2.

\section{REFERENCES}

1. A. A. Albert, Structure of algebras, Amer. Math. Soc. Colloq. Publ., vol. 24, Amer. Math. Soc., Providence, R. I., 1939. MR 1, 99.

2. M. E. Sweedler, Multiplication alteration by two-cocycles, Illinois J. Math. 15 (1971), 302-323. MR 44 \#5348.

3. —_, Purely inseparable algebras, J. Algebra 35 (1975), 342-355. YORK 14853

DEPARTMENT OF MATHEMATICS, CORNELL UNIVERSITY, ITHACA, NEW 